\title{
COLECISTECTOMIA LAPAROSCÓPICA EM PACIENTES DE ALTO RISCO
}

\author{
LAPAROSCOPIC CHOLECYSTECTOMY IN HIGH RISK PATIENTS
}

\author{
Marco Antônio Cezário de Melo, ACBC-PE ${ }^{1}$ \\ Otacílio Francisco Albuquerque ${ }^{2}$ \\ Valéria Gondim ${ }^{3}$
}

\begin{abstract}
RESUMO: Objetivo: Relatar a experiência com a colecistectomia laparoscópica na colecistite aguda, em pacientes de alto risco (ASA 4). Método: De 1982 a 2001 foram realizadas, na Clínica Especializada em Doenças do Aparelho Digestivo (DIGEST), 1507 colecistectomias laparoscópicas, sendo 150 (10\%) em colecistite aguda, dentre as quais $10(0,7 \%)$ em pacientes ASA 4. Resultados: Entre estes 10 pacientes observou-se uma faixa etária elevada com média de 70,9 anos (variando entre 50 e 89 anos), maior frequiência do sexo masculino (60\%), período de internação pré-operatório bastante variável (de três a 22 dias), prevalência elevada de colecistite aguda alitiásica (30\%) e grande freqüência de insuficiência renal (40\%) dentre as doenças associadas. Como complicações de pós-operatório houve uma coleção sub-hepática tratada por drenagem guiada por ultrassonografia; duas infecções de sítio operatório, tratadas pela abertura da pele do portal infectado, e um óbito decorrente da perpetuação do quadro séptico em paciente previamente submetido a colecistostomia percutânea. Não houve necessidade de conversão em nenhum dos pacientes operados. Conclusão: Os autores concluem pela viabilidade do método laparoscópico no tratamento da colecistite aguda em pacientes de alto risco, observando-se algumas estratégias específicas.
\end{abstract}

Descritores: Colecistectomia laparoscópica; Colecistite aguda

\section{INTRODUÇÃO}

Há cerca de 10 anos considerava-se como contra-indicação absoluta da colecistectomia laparoscópica situações como gravidez, cirurgia abdominal prévia, intolerância ao aumento da pressão intra-abdominal, obstrução intestinal, coagulopatia, obesidade, cirrose, intolerância à anestesia geral, coledocolitíase e colecistite aguda ${ }^{1}$. Com o passar dos anos, a maior experiência do cirurgião com o método e o melhor conhecimento e controle das repercussões do pneumoperitônio pelo anestesista, ampliaram as indicações dos procedimentos laparoscópicos e restringiram suas contraindicações. Recentemente a laparoscopia vem sendo utilizada na definição diagnóstica, mesmo em pacientes críticos, com quadro séptico, internados em CTI, sendo realizada, inclusive, à beira do leito ("bedside laparoscopy") 2 .

Desde o início da experiência da DIGEST, em 1992, a colecistectomia laparoscópica foi indicada para o tratamento de todas as patologias cirúrgicas

1. Chefe da Equipe Cirúrgica da Clínica Especializada em Doenças do Aparelho Digestivo. Cirurgião do Serviço de Cirurgia Geral do Hospital das Clinicas da Universidade Federal de Pernambuco (HC - FMUFPE)

2. Cirurgião da Equipe Cirúrgica da Clínica Especializada em Doenças do Aparelho Digestivo. Cirurgião do Hospital da Restauração - SUS - PE.

3. Anestesista da Clínica Especializada em Doenças do Aparelho Digestivo. Cooperada da COOPANEST - PE. 
benignas da vesícula, mesmo na colecistite aguda. $\mathrm{O}$ limite de sua indicação, no entanto, baseava-se no estado físico do paciente de acordo com a classificação da Associação Americana de Anestesia (ASA). Em princípio limitou-se aos pacientes classificados até como ASA 2. A partir de 1996, com a experiência adquirida pela equipe e a introdução de manobras táticas citadas em literatura ${ }^{3}$ e outras elaboradas pela própria equipe, começou-se a intervir em pacientes com colecistite aguda classificados como ASA 4. Este trabalho relata esta experiência em que pacientes de alto risco, foram submetidos à colecistectomia laparoscópica por conta de colecistite aguda litiásica ou alitiásica.

\section{MÉTODO}

Entre 1996 e 2001 foram submetidos à colecistectomia laparoscópica, 10 pacientes considerados como doentes críticos (ASA 4), por apresentarem um quadro séptico decorrente de uma colecistite aguda com descompensação de pelo menos uma doença pré-existente. Todos oriundos de clientela privada, pertencentes a uma classe social definida como A ou B.

Foi utilizada, para a realização da colecistectomia laparoscópica, a técnica americana ${ }^{4}$. Algumas estratégias descritas pela literatura para pacientes de alto risco ${ }^{3} \mathrm{e}$ outras ditadas pela própria experiência dos cirurgiões e anestesistas do grupo foram obedecidas. As variações técnicas e táticas utilizadas neste tipo de paciente estão abaixo relacionadas:

1)- O pneumoperitônio é realizado, preferentemente, pela técnica aberta (Hasson), diminuindo o risco de lesão de alças freqüentemente distendidas nestes pacientes.

2)- Manutenção da pressão intra-abdominal em torno dos $8 \mathrm{mmHg}$ (máximo de $10 \mathrm{mmHg}$ ) já que os pacientes críticos são mais sensíveis á hiperpressão abdominal.

3)- Utilização da ótica de $30^{\circ}$, por facilitar, com sua angulação, a visão das estruturas do pedículo quando da utilização de uma menor pressão intra-abdominal. 4)- Punção sistemática para a evacuação parcial do conteúdo da vesícula (não totalmente) com o fim de facilitar sua apreensão pelos "graspers".

5)- Utilização de "graspers" apropriados ao processo inflamatório. Com dentes para a tração cranial da vesícula e com ramos mais longos para o manuseio do infundíbulo.
6)- Inserção de um portal adicional para tração das alças no sentido caudal colocado na borda externa do músculo reto abdominal esquerdo na altura da cicatriz umbilical.

7)- Dissecção cuidadosa, seccionando-se as aderências bem junto à parede vesicular especialmente na abordagem de seu pedículo (identificação segura do cístico e da artéria cística).

8)- Utilização do ultra-som laparoscópico (no lugar da colangiografia) para avaliar a concomitância de cálculos da via biliar principal (mais rápido e mais seguro, mantendo a mesma sensibilidade e especificidade).

9)- A artéria cística é "clipada" antes da secção do cístico para evitar-se o esgarçamento favorecido pelo processo inflamatório.

10)- O ducto cístico é ligado com um "endoloop" após sua clipagem e secção, para evitar que o "clip" do cístico solte-se após cessar o processo inflamatório. 11)- Utilização de um saco para a colocação da peça cirúrgica (vesícula biliar) no intuito de minimizar a contaminação durante sua retirada através do portal umbilical.

12)- Drenagem sistemática da área operada com o fim de diminuir a ocorrência de coleções abdominais e conseqüentes complicações pulmonares.

Foi utilizado em todos os casos o $\mathrm{CO}_{2}$ para a criação do espaço necessário ao procedimento cirúrgico, sem qualquer tipo de elevador de parede.

\section{RESULTADOS}

Em um total de 1.507 colecistectomias laparoscópicas, foram realizadas $150(10 \%)$ para o tratamento de colecistite aguda, dentre as quais $10(0,7 \%)$ em pacientes classificados como de alto risco.

Na Tabela-1 estão discriminados os principais dados relacionados a estes 10 pacientes, tais como: idade, sexo, período de internação pré-operatória em dias, o diagnóstico da colecistite aguda (litiásica ou alitiásica) e suas complicações pós-operatórias, inclusive óbito. Percebe-se, nos casos analisados, uma faixa etária elevada com média de 70,9 anos (variando entre 50 e 89 anos), uma maior frequiência do sexo masculino $(60 \%)$, um período de internação préoperatório bastante variável (de 3 a 22 dias), uma prevalência elevada de colecistite aguda alitiásica $(30 \%)$ e uma grande frequência de insuficiência renal $(40 \%)$ dentre as doenças associadas, justificada pela vinculação de um dos autores (Albuquerque) a 
Tabela 1 - Experiência com colecistectomia laparoscópica em pacientes de alto risco

\begin{tabular}{|c|c|c|c|c|c|}
\hline $\begin{array}{l}\text { Idade } \\
\text { em anos }\end{array}$ & $\begin{array}{r}\text { Sexo } \\
(\mathbf{M} / \mathbf{F})\end{array}$ & $\begin{array}{l}\text { Intern. pré-op } \\
\text { em dias }\end{array}$ & Diag. & $\begin{array}{l}\text { Diagnósticos } \\
\text { Associados }\end{array}$ & $\begin{array}{l}\text { Complicações } \\
\text { Pós-operatórias }\end{array}$ \\
\hline 50 & M & 6 & CAL & Sepsis Ins. Renal & $\begin{array}{l}\text { Coleção abdominal } \\
\text { + Pneumonia }\end{array}$ \\
\hline 69 & $\mathrm{~F}$ & 5 & CAL & $\begin{array}{l}\text { Sepsis Ins. Respiratória } \\
\text { Ins.Cardíaca }\end{array}$ & \\
\hline 81 & M & 16 & CAL & $\begin{array}{l}\text { Sepsis Ins.Circulatória } \\
\text { Ins. Renal }\end{array}$ & $\begin{array}{l}\text { Manutenção da } \\
\text { sepsis + Óbito }\end{array}$ \\
\hline 63 & M & 3 & CAL & Sepsis Diabetes & \\
\hline 68 & M & 10 & CAA & $\begin{array}{l}\text { Sepsis Ins.Respiratória } \\
\text { Seq. AVC Diabetes }\end{array}$ & \\
\hline 70 & M & 6 & CAL & Sepsis Ins.Renal & \\
\hline 65 & M & 2 & CAL & Sepsis Diabetes & ISO \\
\hline 66 & $\mathrm{~F}$ & 13 & CAA & Sepsis Ins.Renal Diabetes & ISO \\
\hline 89 & $\mathrm{~F}$ & 22 & CAA & Sepsis Ins.Respiratória Diabetes & \\
\hline 88 & $\mathrm{~F}$ & 15 & CAL & Sepsis Ins.Respiratória Seq. AVC & \\
\hline
\end{tabular}

$C A L=$ Colecistite Aguda Litiásica $; C A A=$ Colecistite Aguda Alitiásica $;$ Intern.$=$ Internamento $;$ Pré-op = Pré-Operatório; Diag. $=$ Diagnóstico; Ins. = Insuficiência $;$ Seq. AVC = Seqüela de Acidente Vascular Cerebral; ISO = Infecção de Sítio Operatório

um serviço de hemodiálise. A tração caudal com um afastador tipo "endoflex" fez-se necessária em 6 dos 10 pacientes. Todos os procedimentos foram realizados pela via laparoscópica, sem necessidade de conversão para cirurgia aberta.

O ultra-som laparoscópico só foi utilizado nos três últimos pacientes e a colangiografia trans-operatória deixou de ser realizada pela grande dificuldade técnica decorrente do processo inflamatório local. Nenhum cálculo de colédoco foi identificado neste grupo de pacientes.

Raras extra-sístoles foram observadas em dois pacientes (sem necessidade de drogas para sua correção) e drogas vasoativas foram utilizadas pelo anestesista, durante o ato operatório, para a manutenção do equilíbrio hemodinâmico em um destes pacientes. Todos os operados mantiveram-se em respiração assistida por algumas horas após o procedimento. Como complicações pós-operatórias, observou-se um paciente com coleção sub-hepática de aspecto biliar, puncionada e esvaziada sob orientação de ultra-sonografia que apresentou, concomitantemente, uma atelectasia, seguida de pneumonia na base pulmonar direita.
Outros dois pacientes evoluíram com infecção de sítio operatório superficial, localizada em portal umbilical, tratada pela abertura da pele, com rápida resolução do processo infeccioso. $\mathrm{O}$ paciente previamente submetido a uma colecistostomia percutânea guiada por ultra-sonografia não solucionou seu quadro séptico, mesmo após a realização de colecistectomia laparoscópica, evoluindo para o óbito.

Os dois pacientes com sequela de AVC, usando Varfarina, tiveram esta droga substituída por Enoxaparina até seus parâmentros de coagulação atingirem limites compatíveis ao procedimento.

\section{DISCUSSÃO}

A cirurgia vídeo-laparoscópica tem como característica básica diminuir a agressão e conseqüente trauma cirúrgico. Tem sido demonstrada, nesta abordagem, uma menor repercussão orgânica, representada por menor reação metabólica, inflamatória e imunológica quando comparada a uma cirurgia aberta $^{6}$. Isto representa um grande benefício para o paciente, principalmente àqueles mais graves, já com comprometimento de órgãos e sistemas. 
No entanto, consequências desta abordagem cirúrgica, tais como efeito mecânico do pneumoperitônio, posição do paciente e absorção de $\mathrm{CO}_{2}$, além de algumas alterações circulatórias locais, foram responsáveis pela contra-indicação antes obedecida para os pacientes críticos ou de alto risco ${ }^{7}$.

Apesar dos efeitos hemodinâmicos do pneumoperitônio no choque séptico produzir importantes alterações em alguns parâmetros cardíacos ${ }^{8}$, a cirurgia laparoscópica parece ser um procedimento seguro se estas alterações forem convenientemente tratadas ${ }^{9}$. Em levantamento realizado na literatura analisando-se um total de 100 pacientes críticos submetidos a uma laparoscopia diagnóstica, boa parte efetuada à beira do leito $(40 \%)$, apenas em um único paciente não se conseguiu complementar o procedimento por problemas cardiocirculatórios ${ }^{10}$.

No início dos anos 90, já havia preocupação com as dificuldades técnicas da colecistectomia laparoscópica em paciente com processo inflamatório intenso, sendo publicadas algumas táticas para resolução de diversos problemas relacionados a este tipo de paciente ${ }^{3}$. Baseado nestas afirmativas da literatura e com o amadurecimento da equipe de cirurgiões e anestesistas da DIGEST foi elaborado um protocolo seguido a partir de 1996, quando a colecistectomia laparoscópica passou a ser utilizada, também, para os pacientes de alto risco, classificados como ASA 4.

A utilização da ótica de $30^{\circ}$ e a tração caudal por um afastador tipo "endoflex" parece ter compensado a dificuldade de abordagem do cístico e artéria cística provocada pela baixa pressão utilizada para manutenção do pneumoperitônio, pois todos os procedimentos foram complementados pela via laparoscópica, sem necessidade de conversão para cirurgia aberta. Esta menor pressão (8 a $10 \mathrm{mmHg}$ ) pode ter sido a responsável pela baixa ocorrência de distúrbios circulatórios, pois somente um paciente necessitou de droga vaso-ativa durante o procedimento.

A necessidade de punção, para a evacuação parcial do conteúdo da vesícula, com o fim de facilitar sua apreensão e a utilização de "graspers" com dentes, para a tração cranial da vesícula, e de ramos mais longos, para o manuseio do infundíbulo, parecem condutas óbvias.

A utilização de ligadura adicional do ducto cístico com "endoloop", objetivando evitar o escape pela mobilização do "clip" do cístico no pós-operatório imediato, já sugerido pela literatura ${ }^{3}$, foi introduzido, também, face à ocorrência de um escape de bile pelo cístico em paciente com colecistite aguda, reoperado no $6^{\circ}$. DPO por coleperitônio, quando foi diagnosticada, ainda, uma coledocolitíase residual.

O uso de um saco ("bag") ou mesmo um "dedo de luva" para colocação da peça operatória antes de sua retirada da cavidade abdominal para minimizar a incidência de infecção do sítio cirúrgico é um procedimento clássico na literatura ${ }^{3}$. $\mathrm{Na}$ casuística analisada, mesmo utilizando-se deste artifício técnico, porém, foram observadas duas infecções no portal umbilical, local de retirada de todas as peças cirúrgicas.

Como a literatura tem demonstrado que o tempo de realização do ultra-som na avaliação da via biliar é a metade do gasto com a efetivação da colangiografia trans-peratória, mantendo uma sensibilidade comparável ${ }^{11}$, com o aprendizado na utilização deste novo método diagnóstico e pela evidência, durante a experiência aqui relatada, de uma grande dificuldade de cateterização do cístico face ao processo inflamatório existente, começou-se a utilizar o ultra-som em substituição à colangiografia nos últimos três pacientes.

A drenagem sistemática da área operada foi acrescentada após a verificação de que, nos 60 primeiros casos de colecistite aguda submetidos à cirurgia laparoscópica, foi encontrada uma incidência de 7\% de coleções intra-abdominais e 5\% de complicações pulmonares associadas ${ }^{5}$. Após a utilização da drenagem sistemática observou-se uma queda para $1 \%$ nos últimos 90 casos, não havendo mais complicação pulmonar. Neste levantamento, o caso de coleção intra-abdominal seguido de atelectasia e pneumonia direita, ocorreu antes do uso sistemático da drenagem da área operada.

Com os dados apresentados, conclui-se ser viável a efetivação da colecistectomia laparoscópica em paciente de alto risco, desde que sejam observadas algumas variações técnicas e táticas. Apesar da literatura evidenciar, neste tipo de paciente, um maior índice de conversão, tanto por motivos locais (inflamatórios) como gerais (hemodinâmicos), não houve conversão para cirurgia aberta na casuística mostrada, o que pode ser explicado pelo pequeno número de casos analisados. 


\begin{abstract}
Background: Evolution and experience with laparoscopic cholecystectomy in acute cholecystitis, a better knowledge of alterations provoked by the laparoscopic approach specially related to pneumoperitonium, allowed accomplishment of this procedure in high risk patients (ASA 4), objective of this research. Methods: From 1992 to 2001, 1507 patients underwent laparoscopic cholecystectomy at Clínica Especializada em Doenças do Aparelho Digestivo (DIGEST), 150 (10\%) of them with acute cholecistitis and 10 (0,7\%) ASA 4 patients. Results: Mean age was 70,9 years, varying between 50 and 89 years, and $60 \%$ were males. Preoperative hospitalization varied from 3 to 22 days. There was a $30 \%$ incidence of acute acalculous cholecystitis and 40\% of renal disfunction. Other associated diseases were also observed. Postoperative complications included a sub-liver collection with associated pneumonia, solved by drainage guided by ultrasound, two surgical site infections, and a death due to perpetuation of sepsis on a patient previously submitted to a percutaneus cholecystostomy. None of the patients needed conversion to open surgery. Conclusions: We concluded that the laparoscopic technique approach in the treatment of acute cholecystitis in high risk patients is feasible, since some specific strategies are observed.
\end{abstract}

Key words: Laparoscopic cholecystectomy; Acute cholecystitis; High risk patients.

\section{REFERÊNCIAS}

1. Curet, MJ - Special problems in laparoscopic surgery. Previous abdominal surgery, obesity, and pregnancy. Surg Clin North Am, 2000, 80:1093-1110.

2. Rehm CG - Bedside laparoscopy. Critical Care Clinics, 2000, 16:101-112.

3. Zucker KA, Bailey RW, Flowers J - Laparoscopic management of acute and chronic cholecystitis. Surg Clin North Am, 1992, 72:1045-1067.

4. Reddick EJ, Olsen DO - Laparoscopic laser cholecystectomy: a comparison with mini-lap cholecystectomy. Surg Endosc, 1989, 3:44-48.

5. de Melo MC, Neto JE, Lima LMA, et al. - Colecistectomia laparoscópica na colecistite aguda. Rev Sobracil, 1998; 2:27-31.

6. Hackam DJ, Rostein OD - Host response to laparoscopic surgery: mechanisms and clinical correlates. Can J Surg, 1998, 41(2):103-111.

7. O'Malley C, Cunningham AJ - Physiologic changes during laparoscopic. Anest Clin North Am, 2001, 19:119.
8. Greif WM, Forse RA - Hemodynamic effects of the laparoscopic pneumoperitoneum during sepsis in a porcine endotoxic shock model. Ann Surg, 1998, 227:474-480.

9. Stuttmann R, Vogt C, Eypasch E, Doenh - Hemodynamic changes during laparoscopic cholecystectomy in the high risk patient. Endosc Surg Allied Technol, 1995, 3:174-179.

10. Kelly JJ, Puyana JC, Callery MP et al. - The feasibility and accuracy of diagnostic laparoscopy in the septic ICU patient. Surg Endosc, 2000, 14:617-621.

11. Machi J, Tateishi T, Oishi AJ, et al. - Laparoscopic ultrasonography versus operative cholangiography during laparoscopic colecystectomy: review of the literature and comparison with open intraoperative ultrasonography. J Am Coll Surg, 1999, 188:360-367.

Endereço para correspondência:

Marco Antônio Cezário de Melo

R. Afonso Celso, 66 / 1501- Paranamirim

52060-110 - Recife - PE

E-mail: cezario@truenet.com.br 